\title{
Construire le cadre du jeu : pratiques d'engagement lors de parties de jeux d'édition
}

Jean-Emmanuel Barbier

\section{(2) OpenEdition}

\section{Journals}

Édition électronique

URL : http://journals.openedition.org/sdj/1195

DOI : $10.4000 /$ sdj. 1195

ISSN : 2269-2657

\section{Éditeur}

Laboratoire EXPERICE - Centre de Recherche Interuniversitaire Expérience Ressources Culturelles Education

\section{Référence électronique}

Jean-Emmanuel Barbier, « Construire le cadre du jeu : pratiques d'engagement lors de parties de jeux d'édition », Sciences du jeu [En ligne], 10 | 2018, mis en ligne le 28 octobre 2018, consulté le 30 avril 2019. URL : http://journals.openedition.org/sdj/1195; DOI : 10.4000/sdj.1195

Ce document a été généré automatiquement le 30 avril 2019.

Tous droits réservés 


\title{
Construire le cadre du jeu : pratiques d'engagement lors de parties de jeux d'édition
}

\author{
Jean-Emmanuel Barbier
}

1 Cet article est issu de mon travail de thèse en cours qui aborde la question de l'engagement des joueurs lors des soirées jeux de société organisées par des associations (j'y reviendrai). Le questionnement ci-présent découle de l'observation d'un phénomène récurrent lié à ma présence où je procédais par observation participante en mentionnant ma posture de chercheur. Ma présence, en particulier lors de mes prises de notes ou l'installation d'un dispositif pour filmer les parties, était commentée avec humour par les joueurs. Il n'était pas rare que ces derniers viennent me voir pour signaler une pratique hors du commun, soit en aparté, soit ouvertement en pointant une table ou l'autre. Ces interpellations étaient souvent de même nature : on m'indiquait un joueur particulier qui serait "un cas à analyser", "si tu veux voir un vrai joueur ", une table à observer, car « tu vas voir, ils sont intéressants », "c'est ça l'exploit!». Chacune de ces interpellations marquait un rappel à la norme sociale validant un système de valeurs et d'attentes propres à la rencontre, et à laquelle ma présence offrait sans doute l'opportunité d'une légitimation supplémentaire. Un comportement hors norme devenait ainsi un «cas d'intérêt », et une bonne pratique un « exemple » que je devais reprendre ou décrire pour mes travaux. Par ailleurs, ces apostrophes particulières étaient parfois dépourvus d'explication pouvant justifier a priori l'interjection soudaine, ce qui me mettait face à des situations complexes à interpréter.

2 Chacune de ces prises de paroles traduit une relation particulière entre enquêteur et enquêté ; elle interroge la définition que les joueurs se font des contours d'une partie idéale par l'affirmation d'une légitimité que devrait valider le regard scientifique. L'un des aspects principaux est celui de la gestion de l'engagement ou plutôt des modes d'engagement dans l'activité. Plus que des discours, c'est en effet une série de pratiques, d'attentes et de sens qui sont posés et négociés par les joueurs autour des tables de jeu, ce que Goffman (1974) qualifie de cadre de l'expérience. Je m’appuierai sur les discours et les 
pratiques des joueurs pour questionner les modalités de l'engagement comme maintien du cadre de l'interaction.

3 Après avoir pris en considération les particularités du terrain et des jeux étudiés, une première réflexion se portera sur les discours et les normes produits par les joueurs autour des pratiques d'engagement, en particulier celles qui concernent leurs attentes sur le jeu et les comportements des participants. Ces considérations préalables me permettront ensuite d'éclairer les comportements et procédés mis en place par les joueurs pour gérer le maintien de la rencontre ludique et leur engagement dans l'activité.

\section{Des lieux de rencontre pour joueurs passionnés}

Avant de me lancer dans l'analyse, j'aimerais revenir quelque peu sur mes terrains et ma méthodologie d'enquête. Les données utilisées ici sont issues des terrains d'enquête réalisés pour ma thèse et mon mémoire en anthropologie qui se sont déroulés entre 2007 et 2014, au sein de plusieurs associations organisant des soirées jeux en région parisienne et bruxelloise. Ces soirées jeux sont organisées de manière régulière (hebdomadaires ou mensuelles) et pleinement dédiées à la pratique de jeux de société modernes ${ }^{1}$ pour un public très majoritairement adulte. Afin d'appuyer mon analyse, je me baserai plus particulièrement sur une série d'observations participantes et de parties filmées au Centre National du Jeu (C.N.J.) à Boulogne-Billancourt, accompagnées d'observations provenant d'autres terrains: l'association Strat\&J et les soirées jeux de l'Outpost à Bruxelles. Mon travail de thèse combine une approche ethnographique par observation participante, des entretiens informels et l'exploitation d'un corpus de parties filmées selon la méthode de l'analyse conversationnelle (De Fornel, Léon, 2000).

Concernant les joueurs eux-mêmes, il s'agit de passionnés majoritairement masculins nés entre 1975 et 1985, membres pour la plupart d'une classe moyenne ou supérieure cultivée et citadine. Certains sont très engagés dans les activités " méta-ludiques ", c'est-à-dire de discussion réflexive sur leur loisir ; par exemple, l'un des participants se faisait appeler par son surnom du forum Trictrac.net, principal portail francophone de l'univers ludique dans lequel il s'impliquait beaucoup. Une partie d'entre eux sont aussi des professionnels ou assimilés du champ. J'ai de la sorte pu rencontrer le rédacteur en chef d'une revue spécialisée ainsi que quelques personnes travaillant dans le milieu associatif ou des ludothèques. Certains participants étaient des auteurs ou futurs auteurs de jeux, d'autres des " petits éditeurs ». Seule l'association Strat\&J fonctionnait sur une base différente où les membres fondateurs et les plus réguliers étaient un groupe de quatre amis. Cependant, là aussi, j'observais une implication parallèle aux soirées dans le champ : l'un d'entre eux travaillait pour l'un des premiers bars à jeux de Paris, et une joueuse régulière était employée dans une ludothèque locale.

Dans l'ensemble se dessine une communauté de joueurs très impliquée dans le milieu ludique. Ces joueurs développent des pratiques réflexives, productrices de discours sur le jeu, et certaines de ces soirées jeux étaient organisées par des groupes ayant pour vocation la promotion du jeu comme pratique culturelle. Je reviendrai plus loin sur les conséquences de cet aspect communautaire. 


\section{Jeux en boîte}

7 Dans son texte Du jouet industriel au jouet rationalisé, Brougère décrit les jeux de société et pose la distinction suivante entre jeux traditionnels et jeux d'édition:

Les jeux que nous avons le projet d'étudier appartiennent à un autre type d'insertion sociale [que la diffusion orale traditionnelle ou la diffusion écrite de corpus de règles], la diffusion commerciale, qui n'est pas un caractère extérieur $d u$ jeu, mais intrinsèquement liée à ce qu'il est. Il s'agit de jeux d'édition pourvus d'un " copyright », et donc impossibles à copier, solidaires de leur forme de distribution. D'autre part, et c'est là leur caractère fondamental, leurs règles ne sont pas transmissibles isolément du matériel complexe dont elles donnent les conditions de manipulation. (Brougère, 1979, pp. 200-201)

Cette définition est fondamentale dans la compréhension des jeux de société modernes car elle insiste sur la matérialité et l'inscription dans cette dernière d'une logique de consommation d'un dispositif boîte " prêt-à-jouer ». Si l'on peut nuancer la seconde partie de la citation autour de la transmission des règles, car de nombreux jeux récents et nouvelles pratiques tendent à dissocier la circulation des règles et celle du matériel ${ }^{2}$, il n'en reste pas moins vrai que l'articulation forte entre règle et matériel reste encore aujourd'hui pertinente. La découverte de nouveaux mécanismes et de nouveaux jeux est l'un des principaux attraits de ces soirées revendiqués par les joueurs (les dernières sorties attireront généralement plus de joueurs pourvu qu'elles correspondent à certains critères attendus). Cependant, quelques jeux dits anciens, regroupant une catégorie de jeux âgés d'au moins trois ans jusqu'à des jeux édités dans les années 1970, seront rejoués occasionnellement en formant un corpus qui constituera une série de «classiques $»^{3}$.

Pour devenir un jeu, ce dispositif ${ }^{4}$ doit être réalisé, actualisé par les joueurs. Cette mise en acte d'un jeu de société passe par la construction d'une rencontre sociale particulière : la partie. Goffman, dans son ouvrage Encounters [Rencontres] (1961), pointe cependant que la rencontre sociale générée par une partie de jeu ne se limite pas au seul mouvement des pièces ou des cartes suivant un système de règles. La rencontre ludique comprend en effet un autre espace d'interaction, celui d'une situation de face à face prolongée entre plusieurs individus, et dont les règles, les normes et les attentes dépassent le cadre seul du jeu.

\section{Une communauté de pratique centrée autour d'un discours esthétique}

10 Je l'ai précédemment évoqué, la participation aux soirées est souvent motivée par la volonté de découvrir de nouveaux jeux. Cette approche de la pratique est directement à lier avec la constante expansion du nombre de jeux publiés chaque année, et qui constituent un champ ludique vaste dans lequel les joueurs sont invités à se situer par l'expression de leur goût. Avoir un avis sur les jeux récents donne l'occasion de contribuer à la communauté autour de l'actualité ludique, de rendre manifeste son intérêt pour le loisir au reste du groupe. Pour certains joueurs, ces discussions constituent un intérêt en soi et justifient pour eux leur participation aux soirées jeux. Ce 
discours normatif ou critique sera partiellement partagé par les membres d'une même soirée.

Particulièrement présentes en début et en fin de partie, de telles discussions laissent à chaque joueur l'opportunité d'affirmer ses goûts, d'apprendre et de partager ceux du groupe qui se constitue et se reconstitue à chacun de ces échanges; elles prennent parfois l'allure de critiques construites, reposant sur un vocabulaire partagé et un ensemble de critères précis. Ainsi, les jeux de parcours s'appuyant beaucoup sur le hasard (nous reviendrons sur ce point) sont très largement dépréciés des joueurs qui les utilisent comme figures repoussoir, ou mauvais jeux exemplaires. Dans le même temps, les joueurs marquent chacun leur préférence: si l'un n'aime pas les jeux de pirates où il faut se trahir, l'autre apprécie les jeux calculatoires à gestion de ressources. Il s'agit non seulement du sujet le plus discuté de ces soirées, mais aussi des sites web communautaires où beaucoup de participants $\mathrm{y}$ sont actifs dans les sections avis et les forums dont ils fournissent l'essentiel du contenu. De nombreux joueurs témoignent que la participation à ces discussions est un plaisir recherché, placé au même niveau que la pratique du jeu.

Participer à ces soirées, c'est ainsi prendre part à la production d'un discours collectif sur les jeux au sein de ce que les joueurs appellent la «communauté ludique $»^{5}$ et qui recouvre tout le champ de la pratique des jeux de société moderne. Pour citer Wenger :

J'utilise le concept de réification dans un sens très général pour me référer au processus par lequel nous donnons forme à notre expérience en produisant des objets qui congèlent cette expérience en «chose». En agissant ainsi nous créons des points de focus autour desquels les négociations de sens deviennent organisées. (Wenger, 1998, p. 58, notre traduction)

13 Ici, les joueurs réifient une esthétique des jeux. Il y a une création de points et concepts centraux propres à une communauté à travers des discours partagés ou des manifestations physiques; dans notre cas, il s'agit d'une organisation de la critique et la formation des goûts individuels autour d'une série d'éléments partagés. De plus, il est possible de retrouver une manifestation concrète de cette critique sur les nombreux forums, sites en ligne, journaux spécialisés et blogues produits par la communauté ludique collaborant à une étape supplémentaire de cette réification des discours. Il est important de souligner que chez Wenger, la participation aux activités et la réification produite par le groupe élaborent les deux formes d'engagement dans la communauté de pratique. Autrement dit, en produisant un discours commun, les joueurs contribuent autant à la construction de cette communauté qu'à leur participation aux jeux.

\section{L'esthétique des jeux : l'importance des mécanismes de jeu}

14 Ce discours critique co-construit par les joueurs lors de ces soirées est avant tout un discours esthétique. Il porte principalement sur les mécaniques des jeux, leur intérêt et leur pertinence par rapport au système global qu'elles forment autour d'une échelle de valeurs allant du bon au mauvais jeu. Un bon jeu doit proposer un ensemble cohérent de règles. Celles-ci s'articulent toujours autour d'un dispositif d'inscription du choix des joueurs qui se conclut par un système qui va sanctionner mécaniquement ces choix ${ }^{6}$, en leur donnant un enjeu, c'est-à-dire un sens, une motivation. Caïra (2016) aborde cette question de l'esthétique et de la fiction dans les jeux. Il y distingue trois dimensions de la 
fiction autour desquelles s'articule l'essentiel des jugements esthétiques : la mimétique (thématique du jeu), l'axiomatique (mécanique et règles du jeu) et la simiotique (relation entre simulant et simulé). Ces trois catégories permettent une plus grande finesse de l'analyse des jeux par rapport à une approche traditionnelle de la fiction dans les jeux. Chez Caillois (1958), la confusion entre les différentes formes l'amène à distinguer les jeux fictifs des jeux à règles, et à ne comprendre la fiction dans les jeux que comme séparée du réel.7 Si le jeu est bien un "faire comme si ", une activité métaphorique comme le soulignera Hamayon (2012), il y a une différence entre un faire comme si mimétique (qui place donc le thème au centre) et celui axiomatique (qui nous rapproche plus de l'idée de l'acte de foi décrit par Huizinga [1938] : pour jouer, il faut accepter la validité des règles).

Comme le souligne Caïra (2016), si l'aspect ornemental des jeux est une composante à évaluer, elle n'est pas pour autant centrale dans ce qui constitue le jugement esthétique des joueurs sur les jeux. Ces derniers accordent en effet une plus grande importance aux mécaniques de jeu, à l'aspect axiomatique, en particulier, autour des enjeux qu'il oppose aux joueurs. Par exemple, un jeu réputé difficile ${ }^{8}$ est un enjeu en lui-même pour certains ; il nécessite une plus grande maîtrise du système de jeu et des stratégies adaptées, mais c'est justement l'occasion pour le joueur de démontrer ses compétences. Pour d'autres, la difficulté peut cependant représenter un obstacle. Être performant nécessite un apprentissage et un investissement conséquent, ce qui entraîne un déséquilibre relatif au nombre de parties jouées par chacun. Certains jeux vont alors contrer ce phénomène en augmentant la place du hasard afin de compenser le défaut de compétence individuelle. Un jeu trop dépendant du hasard sera en revanche très critiqué. Si cette limite varie d'un joueur à l'autre, elle évolue autour du même critère : l'agentivité du joueur, c'est-à-dire, sa capacité à être et rester acteur dans le déroulement du jeu.

Le subtil dosage entre complexité des compétences sanctionnées, part de hasard et agentivité, est à l'origine de la grande variabilité des formes de jeu. Ces différents dosages et formes de sanctions forment autant de genres ludiques, différemment appréciés par les joueurs, autour de ce que l'on pourrait appeler une esthétique du fun. Si ces catégories font toujours débat, on retrouve ainsi les party games ${ }^{9}$ qui sont des jeux aux règles simples, rapides à expliquer, à apprendre et à mettre en acte, le plus souvent articulées autour de l'habileté soit expressive soit gestuelle (faire deviner, attraper rapidement un objet); les joueurs leur opposent les jeux experts qui sont longs, complexes et punitifs dans leurs mécaniques, nécessitant quant à eux un apprentissage de stratégies optimales, et donc d'une répétition régulière de parties pour améliorer ses performances de jeux; et enfin une autre catégorie souvent mise en relation par les joueurs avec les deux précédentes : les jeux familiaux qui laissent, eux, une grande part au hasard.

Nous retrouvons l'un des espaces d'engagement du modèle MÉDIAL (Moteur, Épreuve, Diégèse, Intrigue, Autrui, Ludologie), développé dans ce numéro par Caïra (2018) dont j'utiliserai l'apport théorique pour appuyer l'analyse de l'engagement des joueurs. ${ }^{10}$ Ici, la critique des jeux porte sur leurs mécaniques, notamment sur les aspects Moteur (la façon dont le jeu se déroule, la clarté et l'accessibilité de ses règles ainsi que l'affordance du matériel) et d'Épreuve (le jeu est-il porteur d'enjeux pour le joueur? Lui permet-il de se réaliser ?) que l'on retrouve dans le système de sanctions. Les discours critiques me permettent déjà de constater l'importance cruciale accordée par les joueurs à ces deux dimensions qui s'articulent autour de la question de la fiction axiomatique.

De même, la qualité du matériel de jeu et des illustrations sera uniquement pointée si elle est très bonne ou au contraire très mauvaise. Les joueurs évaluent cette capacité d'un jeu 
à livrer ses affordances ${ }^{11}$, ou plutôt lui reprochent son incapacité à le faire. C'est-à-dire, à rendre perceptible pour une majorité des joueurs présents les usages des éléments de jeu relatifs aux règles, et ce quelle que soit la maîtrise et l'expérience préalable du joueur. Un beau matériel renforcera naturellement l'engagement Diégétique (et donc la lisibilité du jeu). Un mauvais matériel risque de faire complètement disparaître l'intérêt pour le thème en ne manifestant pas ce dernier. Dans le pire des cas, il rendra le jeu illisible. Par exemple en créant des confusions entre décors et cases, ou en ne distinguant pas certains éléments de jeux pourtant nécessaires dans des contextes très différents, ce qui est susceptible de limiter l'engagement Moteur ou dans l'Épreuve. Voici quelques déficiences du matériel qui reviennent régulièrement dans les critiques des joueurs : le manque de clarté des règles, l'absence d'aide de jeu, les difficultés à lire le matériel de jeu en raison d'un défaut de contraste, d'une palette de couleurs peu claire ou du tracé des parcours peu évident. Plus le jeu possède des défauts, plus le joueur est susceptible de commettre une erreur d'interprétation sur les choix qui lui sont offerts, ce qui déforcerait à terme son agentivité.

19 Similairement, la thématique d'un jeu sera jugée dans sa capacité à s'articuler aux mécaniques. Pour Caïra (2018), cette articulation se retrouve dans l'aspect simiotique de la fiction. Autrement dit, la Diégèse, cet « enrobage fictionnel », pour citer un joueur, doit favoriser l'intérêt pour les mécaniques et les enjeux du jeu en leur apportant une signification supplémentaire. La partie devient alors une Intrigue qui va évoluer en suivant des rebondissements générés par les actions des joueurs, ce qui renforce l'affordance des règles en leur donnant une signification thématique. Toutefois, l'absence d'une telle dimension thématique ne sera pas jugée dommageable tant que le jeu fournit des règles suffisamment claires, un intérêt ou des enjeux pertinents et justifiés.

\section{Figures de joueur : des normes sociales qui favorisent l'engagement ludique}

Si les discours esthétiques permettent de dégager ce qui, dans le dispositif ludique, renforce l'engagement dans la situation sociale, ce ne sont cependant pas les seuls discours émis par les joueurs sur la question de l'engagement. Les nombreux rappels des normes sociales qui entourent la rencontre ludique permettent eux aussi de mieux saisir les enjeux et valeurs placés par les joueurs sur le déroulement idéal d'une partie et les comportements attendus de la part des participants. J'ai eu l'occasion d'évoquer une forme particulière de ces rappels de la norme dans l'introduction, celle spécifiquement liée à ma présence en tant que chercheur quand les joueurs me pointaient ce qu'ils estimaient pertinent, exemplaire ou déviant selon eux. Ces différents commentaires balisent les attitudes des joueurs dans le champ social des soirées. Ce discours normatif s'articule autour de figures construites par le groupe. Inscrit dans un champ communautaire, il est partagé et débattu, faisant émerger les sempiternels débats entre ce qui est normal ou ce qui ne l'est pas.

21 La première figure, sans doute la plus connue en dehors du champ ludique, est celle du mauvais joueur. Cette figure est directement issue des représentations courantes dans notre société. Le mauvais joueur peut être un mauvais perdant, irascible et hargneux dans la défaite, ou un mauvais gagnant hautain et vantard dans la victoire, ou parfois un peu des deux à la fois. On lui associera bien souvent d'autres comportements jugés négatifs, 
comme le fait de pinailler ou de manipuler les règles, son manque d'entrain à jouer lorsqu'il n'est pas en situation de victoire, etc. Dans le cadre des soirées jeux, ces comportements sont jugés problématiques. On se rapproche en effet de la figure du briseur de jeu selon Huizinga (2011 [1938], p. 29). Cependant, le joueur ne brise pas sciemment l'inlusio ${ }^{12}$ ludique, mais par son excès, il rend sa nature arbitraire explicite. En ayant la victoire absolue pour objectif final du dispositif ludique, il rend manifeste un décalage entre deux attentes coexistantes dans ces rencontres ludiques. Il y a l'attente propre à la partie : la victoire, l'enjeu ludique de ce système de sanctions que nous avons évoqué et qualifié comme un engagement dans l'Épreuve selon Caïra. Et il y a le désir de passer un moment agréable en bonne compagnie, qui s'accompagne des normes sociales usuelles des rencontres focalisées où l'évitement du conflit est préféré, comme l'a montré Goffman dans l'essentiel de ses travaux (en particulier 1961 et 1974). Ce second engagement dans l'activité est qualifié par Caïra dans son modèle en tant qu'engagement pour Autrui. ${ }^{13}$

On retrouve une problématique similaire dans les comportements qualifiés d'antijeu. Pour les joueurs, ce terme désigne les situations où un joueur subvertit l'enjeu ludique pour la victoire aux dépens de l'un ou plusieurs de ses adversaires. La situation la plus typique sur laquelle nous reviendrons, concerne les moments où un joueur, souvent en situation où il a déjà perdu, mais pas systématiquement, va sciemment s'en prendre ou favoriser un autre, altérant ainsi tout l'équilibre du jeu qui présuppose une compétition égale entre tous. Dans ce cas particulier, l'antijeu consiste en un surinvestissement de l'engagement pour Autrui, au détriment de l'enjeu ou Épreuve. Comme le mauvais joueur, ces situations brisent un accord tacite de la rencontre en l'ignorant, cette fois-ci, au bénéfice d'une autre forme d'engagement.

L'antijeu s'inscrit toutefois dans un intermédiaire flou. Si cette pratique est souvent pointée et critiquée, il existe beaucoup de situations où elle sera complètement acceptée. Ainsi, un nouveau joueur présent à une table d'habitués sera préservé par ces derniers en début de partie afin de lui laisser une chance. On peut aussi penser aux nombreuses situations où un joueur se donne un handicap au profit de son adversaire. Parfois, l'antijeu est une méthode pour adapter l'épreuve ludique. Il existe une stratégie légitimement admise par la communauté qui consiste à faciliter la progression d'un joueur ou au contraire, le ralentir si celui-ci est en tête. De la même manière, au risque d'engendrer des remarques négatives et des tensions sociales, il est généralement toléré qu'un joueur privé de toute possibilité de gagner redéfinisse ses objectifs en jeu (je reviendrai sur ce point plus loin). Enfin, si ce changement d'objectif ludique est un choix collectif, ou si cela n'entrave pas le plaisir des joueurs, alors la conduite sera complètement acceptée et souvent distinguée de la notion d'antijeu.

La seconde figure est celle du «bon joueur ». Plus que normative, cette figure est surtout légitimante. Moins manifeste, elle fait rarement l'objet de remarques ouvertes en cours de partie. C'est surtout au travers des remarques qui me furent adressées en tant qu'enquêteur que cette figure m'est devenue accessible. Les participants interrogés rapportent deux qualités nécessaires pour être un bon joueur : l'efficacité et l'affabilité. Le bon joueur gagne souvent aux jeux qui ne dépendent pas du hasard. Il est capable de réaliser de beaux coups ${ }^{14}$, c'est-à-dire, des revirements mémorables en jeu. Être reconnu dans le groupe pour ses victoires ou l'accomplissement de beaux coups certifie en quelque sorte sa capacité à analyser une situation de jeu et à savoir en tirer parti. Le bon joueur 
est aussi celui qui égaie la rencontre ludique par sa rapidité au jeu, ses conseils pertinents et par des traits d'humour qui n'entachent pas son efficacité. Ainsi, le bon joueur est un participant qui ajoute une valeur supplémentaire à l'une ou plusieurs des dimensions de l'engagement, notamment celle de l'Épreuve. Il n'est pas rare de voir des habitués défier le bon joueur à l'un de ses jeux favoris pour tester une nouvelle tactique ou simplement pour le plaisir de se confronter à quelqu'un dont les capacités sont reconnues. C'est de la même façon vers lui qu'ils se tournent lorsqu'ils veulent partager une anecdote ou plaisanter sur un jeu de mots.

Il me reste enfin à évoquer ce que certaines personnes sur mes terrains nommaient «la politesse du joueur ", à savoir la vitesse de jeu. Prendre trop de temps à jouer est tout aussi rédhibitoire que l'irascibilité d'un mauvais joueur, parfois même plus. Cela tient principalement au fait que beaucoup de jeux utilisent un système de tour de jeu, par conséquent la temporalité devient celle des coups; tant qu'un joueur ne joue pas, l'activité est suspendue et l'engagement Moteur collectif est ainsi tributaire de chacun. À cheval entre temporalité discrète des coups et temporalité ordinaire continue, les joueurs entrent dans une attente prolongée avec une suspension excessive de l'intrigue ludique qui les laisse en situation de coprésence tant que l'action ne reprend pas. L'importance accordée par les joueurs à cette dimension s'exprime à travers les nombreuses remarques qui entourent cette question. L'expression «la politesse du joueur» traduit cette importance, et sous-entend qu'il faut " réfléchir pendant le tour des autres joueurs » afin de ne pas les faire attendre durant son tour. Une expression allemande, «ein bisschen schneller bitte [un peu plus vite s'il vous plait]” était ainsi devenue l'emblème d'une communauté de joueurs en ligne (sur le site Brettspielwelt) et l'ont même imprimée sur des t-shirts comme signe distinctif.

La lenteur en jeu s'accompagne aussi de plusieurs connotations sociales. Selon la forme prise par cette lenteur, certains joueurs y dénoteront un manque de compétence ludique, une incapacité à juger rapidement la situation. D'autres y verront un manque d'attention vis-à-vis d'eux, pensant que le fautif a consacré son temps à se tourner les pouces entre ses tours, sans observer la partie. D'une manière générale, on considérera que le joueur lent se prend trop au sérieux, partageant les travers du mauvais joueur. À vouloir être perfectionniste, il n'ose pas prendre les risques qui auraient insufflé une meilleure tension ludique chez les autres joueurs. Ces connotations permettent par ailleurs de comprendre pourquoi la lenteur ne va pas être reprochée dans certaines situations : les joueurs débutants seront plus facilement pardonnés s'ils prennent du temps face aux joueurs expérimentés. Dans ce cas, les échanges seront focalisés sur les conseils tactiques ou le signalement des éléments pertinents du jeu, et bien entendu, ils s'accompagnent du jeu d'influence et de défiance.

Une logique se distingue parmi cette sélection de comportements qui sont socialement sanctionnés par des rappels à la norme. On valorise le comportement d'un joueur capable de maintenir et de favoriser chez autrui l'engagement Moteur dans le jeu et celui de l' Épreuve. À l'inverse, les comportements les plus critiqués manifestent un désengagement dans l'un des aspects de l'activité, que ce soit vis-à-vis du jeu ou des autres joueurs. Ils menacent le cadre de l'interaction puisqu'ils sont à l'origine des situations qui remettent en cause l'inlusio ludique. 


\section{Menaces sur la rencontre : la partie, un cadre d'interaction précaire}

Comme tous les cadres de l'interaction, la situation ludique est particulièrement sensible aux événements perturbateurs comme le signalait Huizinga (2011 [1938]) avec le concept d'Inlusio. Goffman (1991) ne s'est d'ailleurs pas trompé, le point de départ de sa théorie des rencontres focalisées qui le mènera à terme vers sa théorie des cadres de l'expérience, s'appuie sur l'exemple des situations de jeu en ce qu'elles rendent justement plus perceptibles les surgissements du monde extérieur et les difficultés posées pour que les participants maintiennent l'interaction ludique.

Dans la partie de son ouvrage Encounters, nommée fun in games [L'amusant dans les jeux], Goffman (1961) développe une théorie du maintien de l'engagement des participants dans un jeu qu'il étend aux rencontres focalisées en général. Pour les jeux en particulier, ce maintien réside dans la sensation d'euphorie à l'origine du fun in games. Il définit ce concept comme l'ensemble des occasions où les fins, les moyens et les attentes correspondent, lorsque la situation se déroule naturellement avec facilité [ease] et que les ressources propres à la rencontre - comprenant à la fois le dispositif jeu et la dimension d'occasion sociale - sont réalisées. Cette situation d'euphorie sera à l'origine d'un autre concept notable de sa théorie : celui de l'engagement spontané [spontaneous involvement]. Ce concept d'engagement spontané ressemble très fortement à celui d'Inlusio chez Huizinga ou de flow tel que décrit par Csíkszentmihályi (1996), et désigne l'état cognitif d'un participant captivé par l'activité dans laquelle il se trouve. Cette capacité à se concentrer s'y trouve comme démultipliée, ce qui se traduit chez Goffman par une capacité supérieure à ne sélectionner dans la situation que les éléments pertinents et à écarter inconsciemment tout ce qui ne l'est pas.

J'adresserai cependant à ce concept les mêmes limites évoquées par Caïra (2018) au concept de flow dans ce même numéro, à propos de l'impossibilité et de la non-nécessité pour un joueur de se maintenir dans cet état indéfiniment. Pour Goffman (1961), la situation d'engagement spontané est une situation dans laquelle le joueur se retrouve de façon irrégulière au cours de la partie et dans laquelle il va rarement se maintenir de façon prolongée. Le déroulement naturel d'une partie est plus souvent peuplé d'incidents qui vont nuire à l'engagement, soit par des interventions extérieures (surgissement d'événements extérieurs au jeu qui vont perturber le déroulement de la partie) soit par des surgissements propres à la partie ou aux participants.

Ces événements extérieurs constituent une menace parmi tant d'autres envers la situation sociale. Goffman (1961) en fait un élément central de sa théorie, car c'est la résolution de ces conflits par les participants qui permet de comprendre les situations sociales en tant que phénomènes dynamiques. Selon lui, les joueurs mettent en place des procédés qui réagissent aux tensions qu'il nomme dysphorie afin de les distinguer des tensions ludiques qui sont, à l'inverse, un phénomène facilitant l'implication dans l'activité. Cette dysphorie désigne toute situation où le participant éprouve un malaise par un événement - interne ou externe - à tel point qu'il souhaite en sortir. Par exemple, lors d'une partie de poker, lorsque les enjeux deviennent soudainement trop élevés pour le joueur. La dysphorie concerne aussi tout participant qui devient subitement hyperconscient de la situation dans laquelle il se trouve, qui résulte en un détachement 
manifeste. Une situation qui illustre parfaitement cette idée est celle où un joueur s'ennuie et ne s'intéresse plus au développement de la partie.

31 Face à de telles situations, les joueurs vont donc tâcher de maintenir l'engagement dans l'activité par le biais de procédés qui consistent, selon Goffman, à filtrer à travers la membrane de la rencontre ce qui fera partie ou non de la situation. Ce filtrage passe par l'intégration des éléments extérieurs en leur donnant un sens particulier dans le cadre de l'activité, ou bien à les exclure quand ils ne sont pas pertinents en créant un espace secondaire hors du jeu où ces derniers peuvent temporairement s'exprimer ${ }^{15}$. La rareté des interruptions au cours d'une partie témoigne de l'efficacité de ces procédés instaurés par les joueurs. Ces arrêts étaient principalement dus à des raisons extérieures telles que la fermeture du local ou le départ précipité d'un joueur. À une seule occasion la partie fut abandonnée par tous les joueurs à cause d'un jeu jugé mauvais à l'unanimité et que plus personne ne voulait poursuivre.

\section{Gérer l'engagement : choisir sa partie et la mener à son terme}

Alors que les situations d'échec sont rares, celles où une partie sera maintenue à flot malgré les obstacles sont plus courantes. Pour ce faire, les joueurs mobilisent différents procédés. Avant les parties, une grande importance est accordée au choix du jeu. En effet, tous n'ont pas les mêmes attentes ni les mêmes exigences en termes d'intérêt ludique ou encore les mêmes contraintes de temps. J’ai déjà évoqué la façon dont un enjeu trop élevé peut être handicapant pour certains joueurs, et très souvent ces derniers chercheront à équilibrer les niveaux d'une façon ou d'une autre. Chaque jeu possède en outre ses contraintes de temps qui vont déterminer sa sélection afin d'éviter de l'interrompre prématurément.

C'est au moment de la formation des tables que les conséquences sociales sont les plus marquantes pour les mauvais joueurs. S'ils trouvent toujours une table où s'asseoir, les joueurs jugés fautifs seront tout de même évités au maximum par les habitués. Ainsi, j'ai pu observer un participant fraîchement arrivé décliner l'offre d'invitation à une table. Il avait répondu : "Ah non non, je ne veux pas jouer. Je suis venu discuter avec Martin ». ${ }^{16}$ Par la suite, ce joueur vint me confier qu'en réalité, il avait préféré attendre la prochaine partie afin de ne pas se retrouver à la même table qu'un autre participant. Ce dernier était effectivement considéré comme un mauvais joueur, lent à agir durant son tour.

Une fois la partie lancée, les arrangements possibles sont plus limités. On assiste aux mécaniques de transformation/intégration évoquées par Goffman dans Fun in Games : un élément extérieur mentalement impossible à écarter va être traduit en un terme propre au cadre. Par exemple, il n'est pas rare d'observer en fin de soirée, des joueurs qui doivent partir précipitamment pour ne pas manquer le dernier métro. Ils seront, quand c'est possible, soit remplacés par un autre participant soit leurs pièces sont retirées du plateau comme s'ils avaient été éliminés du jeu.

Goffman (1961) mentionne également la mise entre parenthèses du cadre de l'interaction pour autoriser le débordement temporaire d'autres interactions. Si je considère que ce type de mécanisme reste pertinent dans l'analyse d'une situation, les joueurs étudiés adoptent néanmoins une manière plus complexe de gérer ces débordements. Dans les jeux d'édition, la situation ludique dispose de deux temporalités: celle ordinaire et 
continue du temps qui passe, et celle discrète des coups qui font avancer le jeu. Ces deux dimensions entrent régulièrement en conflit. Je viens de parler des joueurs «lents » qui peuvent causer le désengagement et l'ennui chez les autres participants, mais il existe de nombreux jeux où un joueur reste inactif entre deux tours. Or l'attente entre les tours reste incompressible.

Si les joueurs consacrent leur temps d'attente à projeter leur prochain coup et à épier l'action de leurs adversaires, mes observations des tours de paroles démontrent qu'ils utilisent également ce temps de creux pour s'autoriser des apartés. C'est aussi entre les deux tours que l'essentiel de la socialisation des joueurs se produit: on échange les dernières nouvelles avec un ami venu jeter un œil à la partie en cours, on s'assure discrètement que l'on a bien saisi un point de règle, etc. Plus extrême, au $\mathrm{CNJ}$ où il est interdit de manger à une table de jeu, un espace spécifique est dédié à cet usage. Étant donné que les soirées commencent vers $18 \mathrm{~h}$, la plupart des joueurs affamés, engagés sur des parties longues, profitent de cet entre-deux tours pour se précipiter à l'étage et manger quelques bouchées avant de regagner leur place.

Le joueur est néanmoins tenu de garder un œil sur la partie. Il est pour cela aidé par les annonces des autres joueurs, comme autant de rappel que la partie se poursuit. Il n'est en effet pas rare de constater que les joueurs énoncent à voix haute les actions qu'ils accomplissent en usant ces termes-clefs : « lieu historique à six », «je récolte »... La fin d'un tour sera presque systématiquement marquée par des annonces de la part du joueur qui conclut son tour et/ou de celui qui débute le sien. Ces annonces ne sont pas tout le temps spécifiques, il suffit d'un simple « j'ai fini ", « à ton tour ", « à toi » qui peuvent être complétés par un geste de la main ou la transmission d'un objet nécessaire au jeu, tel que les dés. ${ }^{17}$

La gestion des espaces d'engagement apparaît dès lors comme une co-construction des joueurs, en relation directe avec le dispositif et les séquences qu'il impose. Certains jeux, comme Pit (Edgar Cayce, Repos Production, 2008), Dobble (Denis Blanchot, Guillaume Gille-Naves,

Igor Polouchine, Play Factory, 2009), Jungle Speed (Thomas Vuarchex, Pierric Yakovenko, Asmodée, 1996) pour en citer quelques-uns, souvent plus rapides, ne permettent pas de sortir de l'activité alors que pour d'autres, l'attente entre la fin de son tour et le prochain sera plus conséquente. Une hiérarchie dynamique des formes d'engagement va ainsi se mettre en place au cours de la partie, remarquablement visible à travers les prises de paroles réparties entre tous les joueurs. La particularité dans le contexte de cette enquête est l'importance accordée par les joueurs au maintien du déroulement de la partie. L'engagement Moteur tient donc à la fois des règles de ces jeux, du contexte plus général des soirées, pour devenir au final un aspect constituant de la communauté de pratiques qui s'inscrit dans les discours et les normes du groupe.

\section{Les aventuriers de la partie perdue : redonner du sens à son jeu}

La gestion de l'engagement dans la partie passe par la gestion du sens de l'action. En effet, un joueur peut perdre subitement son enjeu dans la partie. Cela advient à chaque fois qu'il découvre que, quelles que soient les actions qu'il va entreprendre, il n'a plus aucun moyen de gagner. Pourtant, le jeu nécessite qu'il continue à jouer puisque la partie est 
toujours en cours et que ses actions continuent à avoir des répercussions pour ses adversaires. Dans cette situation, le joueur certain de ne pas gagner va souvent se redonner un objectif pertinent (c'est-à-dire qu'il peut l'atteindre par les mécanismes du jeu), mais dont la logique se situera hors du système de sanction finale propre à la mécanique du jeu. Il s'agira par exemple de ne pas finir dernier alors que certains joueurs s'amuseront plutôt à perturber la fin du jeu en cherchant à faire gagner un autre joueur à la table ou au contraire à le faire perdre. C'est une situation houleuse car cette redéfinition des objectifs est l'une des formes d'antijeu présentée précédemment, et elle s'accompagne généralement de provocations ou de débats autour de justifications ouvertement arbitraires.

La situation est plus éloquente encore pour les jeux coopératifs quand les joueurs découvrent avant la fin du jeu qu'ils n'ont plus aucune chance de gagner. La situation de dysphorie dans ce cas est beaucoup plus explicite. Alors que paradoxalement le jeu pourrait être considéré comme fini, les joueurs essayeront de le poursuivre jusqu'au bout pour atteindre les conditions de fin de partie usuelles fixées par ce jeu. Le raisonnement souvent donné est la possibilité d'un coup de chance que les joueurs auraient manqué de prévoir. Pour certains, c'est alors l'occasion de tenter les tactiques les plus hasardeuses afin de tester leur efficacité pour les prochaines parties.

41 L'intrigue ludique est elle aussi donnée comme raison de poursuivre le jeu. Même si les joueurs savent qu'ils ont perdu d'avance, ils ne savent pas en revanche comment le jeu va l'emporter. La question n'est plus de savoir s'ils ont gagné ou perdu, mais plutôt de savoir ce qui serait advenu s'ils avaient eu plus de chance ou mieux joué un tour plus tôt. Le nouveau scénario est de savoir si la défaite est due au hasard ou s'ils étaient voués à perdre dès le début parce qu'ils n'avaient pas correctement estimé la menace que représentait une mécanique de jeu. À une occasion, lors d'une partie de Pandémie (Matt Leacock, Z-Man Games, 2008), les joueurs se sont autorisés à tricher suite à une série de malchances lors de la pioche des cartes qui les a menés dans une impasse. Leur but était de vérifier s'ils auraient pu gagner si la pioche leur avait été plus favorable. Leur argument était qu'une telle malchance n'était possible que parce que les cartes avaient été mal mélangées. Puisque le hasard avait été en quelque sorte truqué, ils s'autorisaient donc, eux aussi, à truquer le jeu à leur tour en changeant la dernière carte piochée. Ils emportèrent finalement la partie, tout en reconnaissant qu'ils avaient initialement perdu, et que cette fin de partie était seulement « un substitut de victoire ».

Dans toutes ces situations, les joueurs procèdent à la reconstruction d'une nouvelle forme d'Épreuve lorsque celle proposée par le jeu est rendue inaccessible. Ce changement passe soit par la transformation artificielle de la situation de jeu, soit par l'évaluation d'un potentiel du succès - aurait-il été possible de gagner en poursuivant ma stratégie ? - en épuisant le déroulement du jeu, soit enfin par la redéfinition d'un enjeu à partir des autres sphères d'engagement: l'Intrigue, l'engagement pour Autrui (on veut faire gagner son ami), l'intérêt Ludologique (l'occasion de tester l'efficacité d'une tactique hasardeuse), la Diégèse (puisque je suis tel personnage alors tout naturellement je vais m'en prendre à tel autre). L'objectif commun reste de maintenir le déroulement du jeu pour l'ensemble du groupe, de le réaliser jusqu'au bout et d'accomplir sa dimension autotélique. Ici, la particularité est d'avoir un sens donné à l'activité qui dépasse le seul système de sanctions, mais qui s'accompagne d'un sens nouveau, adjoint par les joueurs en s'appuyant sur d'autres formes d'engagement possibles, et socialement validées, dans l'activité. 


\section{Conclusion}

Les participants des soirées jeux forment ce que l'on peut appeler le cœur de la communauté ludique. Cette communauté de pratique s'est peu à peu construite autour de leur loisir par le biais de rencontres régulières, lors de ce type de soirées ou ailleurs, autour desquelles se constitue un système normé de comportements qui oppose le bon et le mauvais joueur. Toutefois, c'est à la fois la pratique régulière des jeux et la réification de discours sur leur esthétique qui structurent ce groupe. Les critiques esthétiques et les normes sociales s'articulent toutes deux autour de la qualité et de la capacité à engager le joueur dans la partie.

4 La rencontre ludique, qui englobe à la fois les interactions sur le plateau de jeu et l'occasion sociale d'une situation de coprésence autour d'une activité commune, est un cadre d'interaction dont la fragilité potentielle est renforcée par la perte de sens que représente une partie inachevée en raison de la dimension autotélique des jeux. Afin de maintenir la rencontre ludique, les joueurs ont développé différentes stratégies d'engagement. Ces stratégies sont mises en place dès le début de la partie par la sélection de jeux dont ils apprécient les qualités esthétiques, ou par la mise à l'écart de certains joueurs au comportement problématique. Mais surtout, elles sont mises en place pendant la partie à travers la valorisation par les joueurs des comportements qui favorisent l'engagement, et la répartition des modalités de prises de paroles autour des séquences de jeu. Ils savent tirer profit des différences de temporalité entre celles du jeu ou du monde ordinaire, et s'appuient sur un système d'annonces pour maintenir et rendre plus perceptible le déroulement du jeu. Il en résulte un cadre d'interaction en constante négociation entre les participants et l'évolution concrète de la situation; cela en fait un ordre dynamique que les participants cherchent à maintenir ou à transformer.

Les discours normatifs et esthétiques autant que les stratégies de maintien de la rencontre ludique s'articulent autour de logiques d'engagement. Proposé par Caïra dans ce numéro, le modèle MÉDIAL me permet d'apporter un éclairage supplémentaire. Les discours, comme les stratégies, favorisent principalement ce que Caïra désigne par les engagements Moteur et d'Épreuve. Ces deux engagements sont en effet nécessaires au déroulement de la rencontre; rompre trop longuement l'un des deux éléments fait prendre le risque aux joueurs de créer un état dysphorique, de perte de l'inlusio, de sens porté à l'activité. L'engagement pour autrui, valorisé également par les joueurs, est lié à cette situation de coprésence qui s'accompagne de normes comportementales, de respect et d'attention à l'autre que l'on retrouve dans l'idée du fair-play, ou de son opposé lorsque cet engagement est absent (la figure du mauvais joueur).

Les autres formes d'engagement du modèle MÉDIAL permettent aussi de comprendre comment certaines stratégies renforcent les engagements principaux. Ainsi, la Diégèse va consolider les affordances des mécaniques (ou relatives à la règle) d'un jeu et donc faciliter sa prise en main et sa mise en acte via son aspect Moteur par les joueurs. Les stratégies des joueurs suivent des principes similaires, l'humour est par exemple un trait qui met en avant une situation. Lorsqu'un joueur en fin de partie est privé de l'enjeu ludique car il n'a plus de moyens de gagner, la transformation de son rôle (ou de ses objectifs) suit les contours de ces autres formes d'engagement soit pour l'intérêt ludologique de la recherche de nouvelles tactiques, soit le défi de faire perdre le joueur en tête ou d'aider son ami à gagner, caractéristique de l'engagement pour Autrui. 
Les pratiques et les enjeux se retrouvent, là encore, intégrés au discours du groupe qui va articuler autour de ces questions un catalogue de figures normatives et d'attentes envers les jeux et les joueurs. Cette double dynamique va alors peu à peu légitimer les comportements bénéfiques aux rencontres ludiques et la participation à cet effort collectif d'une production commune du discours sur le jeu.

\section{BIBLIOGRAPHIE}

AKRICH M., CALLON M. et LATOUR B. (2006), Sociologie de la traduction : textes fondateurs, Paris, Presses des Mines http://books.openedition.org/pressesmines/1181.

BROUGÈRE G. (1979), « Du jouet industriel au jouet rationalisé », in R. Jaulin (dir.) Jeux et Jouets. Essai d'ethnotechnologie, Paris, Aubier, pp. 193-223.

CAILLOIS R. (1967), Les jeux et les hommes : le masque et le vertige, Paris, Gallimard.

CAÏRA O. (2016) « Théorie de la fiction et esthétique des jeux », Sciences du jeu, n 6, https:// journals.openedition.org/sdj/671

CAÏRA O. (2018), « Les dimensions multiples de l'engagement ludique », Sciences du jeu, n 10, https://journals.openedition.org/sdj/

CALLEJA G. (2011), In-Game : from immersion to incorporation, Cambridge, The MIT press.

CSÍKSZENTMIHÁLYI M. (1996), Creativity: flow and the Psychology of Discovery and Invention. New York, Harper Perennial.

DE FORNEL M., LÉON J. (2000), « L'analyse de conversation, de l'ethnométhodologie à la linguistique interactionnelle. » In Histoire Épistémologie Langage, tome 22, fascicule 1, Horizons de la grammaire alexandrine, pp. 131-155.

DE KOVEN B. (2013), The well-played game: a player's philosophy, Cambridge, The MIT press.

GIBSON J. J. (1977), « The Theory of Affordances », in R. Shaw, J. Bransford (eds) Perceiving, Acting, and Knowing, Hillsdale, Lawrence Erlbaum Associates, pp. 67-82

GOFFMAN E. (1961), Encounters: Two Studies in the Sociology of Interaction - Fun in Games \& Role Distance, Indianapolis, Bobbs-Merrill.

GOFFMAN E. (1991 [1974]), Les cadres de l'expérience, Paris, Editions de Minuit.

HAMAYON R. (2012), Jouer. Une étude anthropologique, Paris, La Découverte/Revue du MAUSS.

HUIZINGA J. (2011 [1938]), Homo ludens, essai sur la fonction sociale du jeu, Paris, Gallimard.

KAPP S. (2013) L'immersion fictionnelle collaborative. Une étude de la posture d'engagement dans les jeux de rôles grandeur nature. Thèse de doctorat, EHESS - Université Libre de Bruxelles.

WENGER E. (1998), Communities of practice: learning, meaning and identity, Cambridge, Cambridge University Press. 


\section{NOTES}

1. Termes courants utilisés depuis peu dans le milieu des joueurs pour désigner leur objet de pratique. D'autres dénominations existent, toutes plus ou moins controversées comme jeux de plateau, jeux d'édition, jeux d'auteur, etc.

2. On songera notamment aux jeux print and play, à la transformation locale de règles, à la transmission orale des règles par l'intermédiaire de la vidéo, etc. Ces pratiques sont autant de cas liminaux qui n'existaient pas au moment de la publication du texte de Brougère.

3. Parmi lesquels on trouvera Puerto Rico (Andreas Seyfarth, Tilsit, 2002), Diplomacy (Alan B Calhamer, 1959), Carcassonne (Klaus-Jürgen Wrede, Hans im Glück, 2000), les colons de Catane (Klaus Teuber, Jeux Descartes, 1995) pour ne citer que les plus célèbres.

4. Pour cette notion, je suis ici l'approche de la sociologie de la traduction et en particulier les travaux d'Akrich, Callon et Latour (2006). Dans cette approche, le dispositif est constitué par la mise en place d'un système socio-technique conçu autour d'un script, qui est acteur de la situation, mais dont l'usage se fait dans la négociation en situation. Ici, le dispositif est constitué par l'ensemble matériel-règle du jeu, le script est en grande partie donné par les règles qui fournissent les conditions de manipulation des éléments (matériels ou non) du jeu.

5. Il existe d'autres communautés ludiques liées aux différentes formes du jeu, en particulier dans le monde du jeu vidéo. Cependant, pour des raisons de simplicité, le terme de communauté ludique recouvre uniquement dans mon présent article celle construite autour des jeux de société modernes.

6. Le format le plus classique et le plus évident à saisir est celui de la piste de score. Les joueurs vont au cours de la partie faire des actions dont certaines vont résulter en un nombre de points à ajouter à leur score représenté par un pion sur une piste numérotée. En fin de jeu, le joueur le plus élevé sur cette piste l'emporte.

7. Il va jusqu'à opposer le jeu au réel via son usage du terme fictif plutôt que celui de fictionnel.

8. C'est-à-dire bien souvent un jeu comportant une grande richesse mécanique, mais où la moindre erreur tactique aura des conséquences durables dans le système de score. Autrement dit, pour être certain de l'emporter, le joueur n'a pas le droit à l'erreur.

9. Souvent traduit en français par "jeux d'ambiance ", même si l'usage de ces termes diverge parfois.

10. Afin de clarifier l'usage du modèle, en particulier lorsque les mots utilisés peuvent provoquer une confusion avec leur usage courant, les références à ce dernier seront indiquées en italique.

11. J'entends ici par affordance les possibilités d'actions offertes par un élément dans une situation, que je reprends aux travaux de Gibson (1975) et dont je retiens l'aspect situationnel et interactif entre acteurs-objets-environnement. Dans le contexte présenté ici, la règle rend possible une quantité limitée d'action face à des situations données et dont la maîtrise et la compréhension constituent l'un des enjeux ludiques : pour faire un choix efficace, il faut en saisir toutes les possibilités. La diégèse proposée par un jeu permet aux acteurs, par exemple, une meilleure saisie des possibilités en proposant des logiques métaphoriques et fictionnelles qui accompagnent et rendent plus facilement perceptibles celles mécaniques.

12. Littéralement « entrée dans le jeu ».

13. Notons que dans d'autres cadres que celui de la soirée, la balance entre les deux formes d'engagement peut être différente. Ainsi, on attend bien souvent des sportifs dans une compétition un engagement bien plus important et manifeste dans l'Épreuve.

14. Nous retrouvons cette idée analysée plus longuement au cœur de la question des jeux bien joués ( well played games) par De Koven (2013). 
15. Dans sa thèse, Kapp (2013) observe un phénomène similaire chez les joueurs de jeu de rôle grandeur nature, en particulier autour de la gestion des moments Time-in et Time-out, et la réflexion de sa seconde partie «Interagir au sein d'un univers. Repousser les limites et renforcer le cadre » où il s'intéresse aux deux tâches de "l'immergé » d'apparence paradoxale : il doit à la fois se plonger dans l'activité et y prendre part, et de l'autre participer à la construction et au maintien collectif du monde fictionnel.

16. Le nom du joueur a été modifié.

17. Notons au passage que ces objets transférés doivent être nécessaires au tour d'un joueur. Car à l'inverse, c'est souvent dans ces moments de transition que des objets non nécessaires et moins systématiques dans les séquences d'action - par exemple un jeton premier joueur, ou le marqueur de tours, etc. - peuvent être oubliés.

\section{RÉSUMÉS}

Les participants des soirées jeux de société placent souvent la rencontre sociale proposée par ces jeux comme l'une des raisons de leur venue. Pour autant, le discours normatif des joueurs laisse paraître une série de facteurs qui risquent de faire échouer l'interaction ludique. On rejoint ici l'argument de Goffman (1961) qui démontre que derrière la solidité apparente des rencontres sociales se cachent des stratégies de maintien d'une situation par ses participants. Après avoir dégagé les particularités de ces soirées jeux, je propose ici d'étudier ces stratégies mises en place par les joueurs pour réussir à maintenir le cadre de la partie. Pour cela, je m'appuierai sur les discours normatifs produits et partagés par les joueurs, puis sur des pratiques mises en place par ceux-ci pour faciliter ou maintenir leur engagement ludique.

Boardgame evenings participants often consider the social pleasure of the encounter one of the main appeal of boardgames. Yet, they let it be understood, through their dialogue, that the social interaction of the aforementioned evenings is not guaranteed to succeed. This fact is confirmed by Goffman's approach (1961), which demonstrates that the strength of social interaction mainly relies upon the strategies used by participants to maintain it. In this article, I set as a goal to study the strategies implemented by the players in order to maintain their involvement throughout the game. To accomplish this, I will first rely on the normative discourse produced by the players on games and expected social behaviours; then I will study the different practices they used to maintain and foster their participant's involvement.

\section{INDEX}

Keywords : frame analysis, encounters, board game, artifact, communities of practices, involvement

Mots-clés : cadres de l'interaction, jeu de société, dispositif, communauté de pratique, engagement 
AUTEUR

JEAN-EMMANUEL BARBIER

Institut Marcel Mauss, Ecole des Hautes Etudes en Sciences Sociales 